\title{
Simulação e modelagem computacionais no auxílio à aprendizagem significativa de conceitos básicos de eletricidade: Parte I - circuitos elétricos simples \\ (Computational modelling and simulation activities to help the meaningful learning of electricity basic concepts: Part I - simple electric circuits)
}

\author{
Pedro F.T. Dorneles ${ }^{1}$, Ives S. Araujo e Eliane A. Veit \\ Instituto de Física, Universidade Federal do Rio Grande do Sul, Porto Alegre, RS, Brasil \\ Recebido em 11/7/2005; Revisado em 21/3/2006; Aceito em 25/9/2006
}

\begin{abstract}
Neste artigo analisamos diferentes concepções e raciocínios dos alunos sobre conceitos básicos envolvidos em circuitos elétricos simples, que se tornam obstáculos para a aprendizagem, e apresentamos um produto de um trabalho de pesquisa, que consiste em um conjunto de atividades de simulação e modelagem computacionais, desenvolvidas com o software Modellus, levando em conta tais dificuldades, com o objetivo de auxiliar o aluno a superá-las.
\end{abstract}

Palavras-chave: simulação computacional, modelagem computacional, concepções alternativas, circuitos elétricos, ensino de Física.

In this paper we analyze different student's conceptions and reasonings regarding basic concepts involved in simple electric circuits, that become obstacles for the learning, and present a product of a research work, that consists in a set of computational modeling and simulation activities, developed with the software Modellus, taking into account such difficulties to help students to surpass them.

Keywords: computational modelling, computational simulation, misconceptions, electric circuits, physics instruction.

\section{Introdução}

Este artigo está embasado em um trabalho de pesquisa $^{2}$, inserido em uma linha que investiga o uso de tecnologias computacionais como recurso instrucional à aprendizagem de Física. Em tal linha de pesquisa, pretende-se apresentar propostas de atividades computacionais que, levando em conta as dificuldades de aprendizagem apresentadas pelos alunos em áreas específicas da Física, possam auxiliá-los a superá-las. Tais propostas abarcam tanto o conteúdo específico, abordado no material instrucional, quanto a metodologia de trabalho, de modo a propiciar condições favoráveis à aprendizagem significativa, no referencial de Ausubel [1, 2].

O primeiro trabalho envolvendo simulação e modelagem computacionais desenvolvido no âmbito desta

\footnotetext{
${ }^{1}$ E-mail: pdorneles@if.ufrgs.br.

${ }^{2}$ Trabalho de mestrado de um dos autores (P.F.T.D.).

${ }^{3}$ Disponível em: http://www.if.ufrgs.br/gpef/graficos_cinematica.zip.

${ }^{4}$ Disponível em: http://www.if.ufrgs.br/gpef/tugk.zip.

linha de pesquisa [3] abordou as dificuldades de aprendizagem dos alunos na interpretação de gráficos da cinemática e teve como subprodutos: i) um conjunto de atividades de simulação e modelagem computacionais ${ }^{3}$, complementares às atividades tradicionais de ensino da cinemática, e ii) a adaptação e validação de um teste ${ }^{4}$ sobre o entendimento de gráficos da cinemática [4].

No presente trabalho, o conteúdo de estudo são circuitos elétricos. Pesquisas recentes relativas à aprendizagem de eletricidade não têm se restringido à identificação de dificuldades dos alunos, mas também apresentam propostas didáticas para a superação das mesmas, e.g., $[5,6]$. Nosso trabalho se alinha com esta abordagem, consistindo em uma proposta didática que envolve o uso de simulação e modelagem computacionais, especificamente com o software Modellus no ensino 
de circuitos simples, constituídos somente por baterias e dispositivos resistivos, e de circuitos RLC.

Neste artigo temos como objetivo divulgar o material instrucional elaborado, após uma ampla revisão da literatura sobre o ensino de circuitos elétricos simples. Iniciamos na seção 2 discutindo estudos referentes a dificuldades de aprendizagem [5-7]. Na seção 3, argumentamos sobre a motivação para o uso de simulação e modelagem computacionais, especialmente com o software Modellus. Os objetivos a serem atingidos pelos alunos ao desenvolverem as atividades computacionais são apresentados na seção 4. Nela também ilustramos as atividades propostas, com três exemplos. Todas as atividades propostas estão disponíveis na Internet [8]. A seção 5 é dedicada à apresentação de nossa estratégia de ensino que envolve além das atividades computacionais um método colaborativo presencial [9] e o método POE (predizer, observar e explicar) [10]. Algumas considerações finais são apresentadas na seção 6 .

\section{Dificuldades de aprendizagem em circuitos elétricos simples}

A eletricidade é uma das áreas da Física que possuem mais estudos referentes a dificuldades de aprendizagem. Estes estudos incluem dificuldades conceituais, concepções alternativas, uso indiscriminado da linguagem e raciocínios errôneos que os alunos costumam apresentar no estudo de circuitos elétricos simples. Em nossa revisão da literatura [11], realizada em nove revistas especializadas em ensino de Física, encontramos 50 publicações sobre circuitos elétricos simples e RLC, nos últimos 20 anos. Dentre as 44 relativas a circuitos simples de corrente contínua, destacamos três: Duit e Von Rhöneck [5], que em 1998 dedicaram um capítulo de um livro ${ }^{5}$ para uma ampla revisão da literatura referente às dificuldades de aprendizagem dos alunos; Shaffer e McDermott [6], que desenvolveram um exaustivo estudo de identificação de dificuldades ${ }^{6}$ e Engelhart e Beichner [7], que desenvolveram um teste com 29 questões para detectar e interpretar conceitos sobre circuitos resistivos de corrente contínua (DIRECT - Determining and Interpreting Resistive Electric Circuit Concepts Test), testado com centenas ${ }^{7}$ de alunos de nível médio e universitário nos Estados Unidos e Ca- nadá, cuja aplicação reproduziu dificuldades relatadas na literatura.

\subsection{Dificuldades conceituais e concepções al- ternativas}

Na Tabela 1 apresentamos as principais concepções alternativas e uma síntese das dificuldades de natureza específicas relacionadas aos conceitos de corrente elétrica, diferença de potencial e resistência elétrica, apontadas nas Refs. [5-7].

A seguir, serão exemplificadas as principais dificuldades conceituais contidas nesta tabela, ilustrando com questões típicas aplicadas aos alunos para identificar suas dificuldades conceituais e concepções alternativas sobre os conceitos físicos envolvidos em circuitos elétricos simples.

a) Dificuldades relacionadas ao conceito de corrente elétrica

Muitos alunos não compreendem que a intensidade da corrente elétrica em um circuito depende não somente das características da fonte, mas também da resistência equivalente do que foi acoplado entre os terminais da fonte (dificuldade 1.1 da Tabela 1). Uma questão típica para identificar este tipo de dificuldade é apresentada por Shaffer e McDermott [6]: solicitados a ordenar o brilho das lâmpadas ${ }^{8}$ presentes nos circuitos 1, 2 e 3 mostrados na Fig. 1, somente $10 \%$ a $15 \%$ dos alunos fornecem a resposta correta: $L_{1}=L_{5}=L_{4}>$ $L_{2}=L_{3}$. Muitos alunos predizem que $L_{2}$ brilha mais do que $L_{3}$ no Circuito 2 da Fig. 1, ou seja, acreditam que a corrente elétrica é consumida no circuito (dificuldade 1.2). Outros tantos acreditam que a ordem dos elementos é relevante (dificuldade 1.3). Por exemplo, há aluno que pensa que todas as lâmpadas $L_{1}, L_{2}, L_{4} \mathrm{e}$ $L_{5}$ têm um mesmo brilho, "porque a corrente está livre para passar por elas sem ter de passar por nenhuma outra lâmpada antes". Este aluno, claramente pensa que as intensidades de $L_{2}$ e $L_{3}$ não são as mesmas. A concepção de que uma bateria é uma fonte constante de corrente elétrica transparece quando os alunos, ignorando o papel da resistência equivalente sobre a corrente no circuito dizem, em relação aos circuitos da Fig. 1, afirmativas do tipo: " $L_{1}, L_{2}$ e $L_{3}$ têm o mesmo brilho, que é maior do que o de $L_{4}$ e $L_{5}$, que são iguais entre si. A mesma corrente elétrica $i$ que passa em $L_{1}$ se divide no Circuito 3 entre as lâmpadas $L_{4}$ e $L_{5}$ ".

\footnotetext{
${ }^{5}$ Learning and understanding key concepts of electricity, in Connecting Research in Physics Education with Teacher Education, An I.C.P.E. Book, (c) International Commission on Physics Education 1997, 1998.

6 Estes autores fazem parte do grupo de Ensino de Física da Universidade de Washington, o qual tem se dedicado a identificar dificuldades dos alunos em vários domínios da Física e as consideram no planejamento de estratégias instrutivas.

7 A primeira versão do teste foi aplicada a 1135 alunos e a segunda a 695.

8 Em todas as questões apresentadas neste artigo admite-se que: as lâmpadas são iguais, os brilhos das lâmpadas crescem quando a intensidade da corrente elétrica aumenta, as baterias são ideais e os fios elétricos possuem resistência elétrica desprezível.
} 
Tabela 1 - Síntese das dificuldades conceituais e concepções alternativas identificadas nas Refs. [5-7].

\begin{tabular}{|c|c|c|}
\hline Conceitos & Dificuldades conceituais & Concepções alternativas: os alunos... \\
\hline 1. Corrente elétrica & $\begin{array}{l}\text { 1. Compreender que a intensidade da corren- } \\
\text { te elétrica em um circuito depende das carac- } \\
\text { terísticas da fonte, mas também da resistência } \\
\text { equivalente do que foi acoplado entre os seus ter- } \\
\text { minais. } \\
\text { 2. Considerar a conservação espacial da corrente } \\
\text { elétrica. } \\
\text { 3. Reconhecer que a intensidade da corrente } \\
\text { elétrica não depende da ordem em que se encon- } \\
\text { tram os elementos no circuito e nem do sentido } \\
\text { da corrente. }\end{array}$ & $\begin{array}{l}\text { a) ... pensam que a bateria é uma fonte de corrente } \\
\text { elétrica constante [5-7]. } \\
\text { b) ... pensam que a corrente se desgasta ao passar por } \\
\text { uma resistência elétrica [5-7]. } \\
\text { c) ... acreditam que a ordem dos elementos no circuito } \\
\text { e o sentido da corrente elétrica são relevantes [5-7]. } \\
\text { d) ... pressupõem que a fonte fornece os portadores de } \\
\text { carga responsáveis pela corrente elétrica no circuito } \\
{[7] \text {. }}\end{array}$ \\
\hline $\begin{array}{l}\text { 2. Diferença de } \\
\text { potencial }\end{array}$ & $\begin{array}{l}\text { 1. Dificuldades em diferenciar os conceitos: dife- } \\
\text { rença de potencial e corrente elétrica. } \\
\text { 2. Dificuldades em diferenciar os conceitos de di- } \\
\text { ferença de potencial e de potencial elétrico. } \\
\text { 3. Deficiência para reconhecer que uma bateria } \\
\text { ideal mantém uma diferença de potencial cons- } \\
\text { tante entre seus terminais. } \\
\text { 4. Calcular a diferença de potencial entre pares } \\
\text { de pontos ao longo do circuito. }\end{array}$ & $\begin{array}{l}\text { e) ...pensam que a bateria é uma fonte de corrente } \\
\text { elétrica constante e não como uma fonte de diferença } \\
\text { de potencial constante [5-7]. } \\
\text { f) ...percebem a diferença de potencial como uma pro- } \\
\text { priedade da corrente elétrica [7]. } \\
\text { g) ... consideram que as diferenças de potencial entre } \\
\text { pares de pontos ao longo do circuito permanecem } \\
\text { constantes [5]. } \\
\text { h) ...associam o brilho de uma lâmpada com o valor } \\
\text { do potencial em um dos terminais da lâmpada [6]. }\end{array}$ \\
\hline 3. Resistência elétrica & $\begin{array}{l}\text { 1. Dificuldades para distinguir resistência equi- } \\
\text { valente de uma parte do circuito e a resistência } \\
\text { elétrica de um elemento individual. } \\
\text { 2. Perceber que a resistência equivalente é uma } \\
\text { abstração útil para obter a corrente total ou a } \\
\text { diferença de potencial em uma parte do circuito. } \\
\text { 3. Compreender que as divisões de correntes } \\
\text { elétrica em um ponto de junção do circuito de- } \\
\text { pendem da configuração do circuito. } \\
\text { 4. Entendimento da associação em série de resis- } \\
\text { tores como um impedimento à passagem de cor- } \\
\text { rente; e da associação em paralelo como um ca- } \\
\text { minho alternativo, para a passagem de corrente. } \\
\text { 5. Identificar associações em série e em paralelo. }\end{array}$ & $\begin{array}{l}\text { i) ...freqüentemente pensam na resistência equivalente } \\
\text { no circuito como se fosse uma propriedade de um ele- } \\
\text { mento individual do circuito [6]. } \\
\text { j) ... ao determinar como se divide a corrente elétrica } \\
\text { em ramos paralelos de um circuito, consideram so- } \\
\text { mente o número de ramos e não as resistências } \\
\text { elétricas relativas dos vários ramos [5- } 7] \text {. } \\
\text { k) ...pensam que se um resistor reduz a corrente por } \\
\text { x, dois resistores vão reduzi-la por } 2 \mathrm{x} \text {, independente- } \\
\text { mente do arranjo dos resistores [7]. } \\
\text { l) ...consideram que resistores alinhados em série estão } \\
\text { associados em série quer haja uma junção ou não entre } \\
\text { eles e que resistores alinhados geometricamente em pa- } \\
\text { ralelo estão associados em paralelo mesmo se há uma } \\
\text { bateria no ramo [7]. }\end{array}$ \\
\hline
\end{tabular}

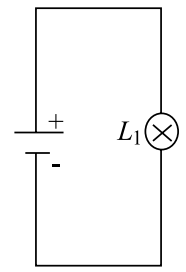

Circuito 1

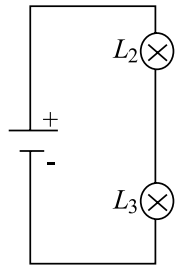

Circuito 2

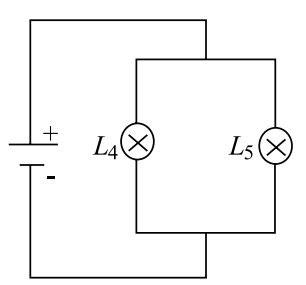

Circuito 3
Figura 1 - Teste típico para verificar se os alunos possuem concepções alternativas sobre corrente elétrica e diferença de potencial $[6]$.

b) Dificuldades relacionadas ao conceito de diferença de potencial

Muitos alunos não compreendem bem os conceitos de potencial elétrico, diferença de potencial e corrente elétrica (dificuldades 2.1 e 2.2). Por exemplo, em relação à confusão entre potencial e diferença de potencial, mais da metade dos alunos não foi capaz de ordenar corretamente o brilho das lâmpadas da Fig. 2, quando o interruptor está fechado, nem responder corretamente o que aconteceria com o brilho da lâmpada

\footnotetext{
9 Terminais para conexão de um elemento do circuito.
}

$L_{1}$ se o interruptor fosse aberto [6]. Pode-se chegar à resposta correta $L_{1}=L_{4}>L_{2}$ e $L_{3}$ observando que a corrente elétrica que passa pelas lâmpadas $L_{1}$ e $L_{4}$ é a mesma e maior do que a que passa por $L_{2}$ e $L_{3}$; ou pode-se raciocinar que a diferença de potencial entre os bornes $^{9}$ de $L_{1}$ e de $L_{4}$ é maior do que a estabelecida entre os bornes de $L_{2}$ e de $L_{3}$.

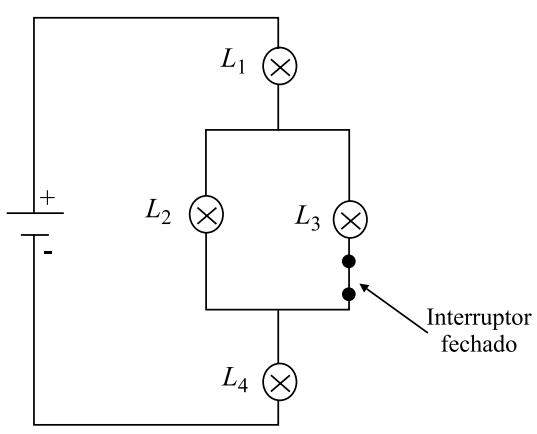

Figura 2 - Teste típico para verificar se os alunos possuem concepções alternativas sobre corrente elétrica e diferença de potencial $[6]$. 
Dificuldades com o conceito de diferença de potencial fazem com que os alunos considerem uma bateria como uma fonte de corrente elétrica constante e não como uma fonte de diferença de potencial constante, conforme já abordado no item anterior (dificuldade 2.3).

A falta de clareza quanto à diferença de potencial, conforme salientam Shaffer e McDermott [6], também faz com que os alunos tenham grandes dificuldades em diferenciar entre:

- uma associação em paralelo conectada diretamente a uma bateria ideal (Circuito 3 da Fig. 1), de modo que a alteração em um dos ramos não afeta o outro, pois a bateria estabelece uma mesma diferença de potencial entre as junções destes ramos

e

- uma associação do tipo da Fig. 2, em que os ramos paralelos não se conectam diretamente à bateria, e a alteração em um dos ramos afeta o outro. Isto é equivalente ao que ocorre quando se tem uma associação em paralelo conectada a uma bateria real.

Em torno de $40 \%$ dos alunos que foram questionados por Duit e Von Rhöneck [5] sobre o comportamento da diferença de potencial entre todos os pares de pontos da Fig. 3 (dificuldade 2.4) concluíram que em todos os casos seria de $6 \mathrm{~V}$. Também não foram capazes de diferenciar os conceitos de diferença de potencial e de corrente elétrica (dificuldade 2.1) nesta situação.

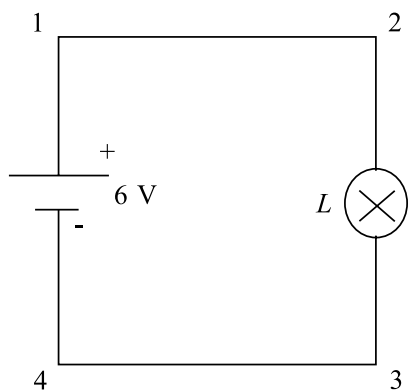

Figura 3 - Representação de um circuito elétrico com uma lâmpada submetida a uma diferença de potencial fixa, para verificar a compreensão do comportamento da diferença de potencial ao longo do circuito [5].

c) Dificuldades relacionadas com conceito de resistência elétrica

Alguns alunos pensam na resistência equivalente de uma parte do circuito como se fosse uma propriedade de um elemento individual (dificuldade 3.1), não percebendo a resistência equivalente como uma abstração útil para obter a corrente total ou a diferença de potencial em uma parte do circuito (dificuldade 3.2). Muitos não são capazes de chegar a uma solução qualitativa correta, embora resolvam com sucesso problemas complicados usando a lei de Ohm e as leis de Kirchhoff. Um exemplo típico em que isto ocorre é nos exercícios sobre brilhos de lâmpadas, como o da Fig. 4. Segundo Shaffer e McDermott [6], muitos alunos não assimilam os conceitos básicos de eletricidade de um modo coerente e não têm condições de elaborar um raciocínio físico, precisando necessariamente se valer de fórmulas ou da intuição, ou ambos.

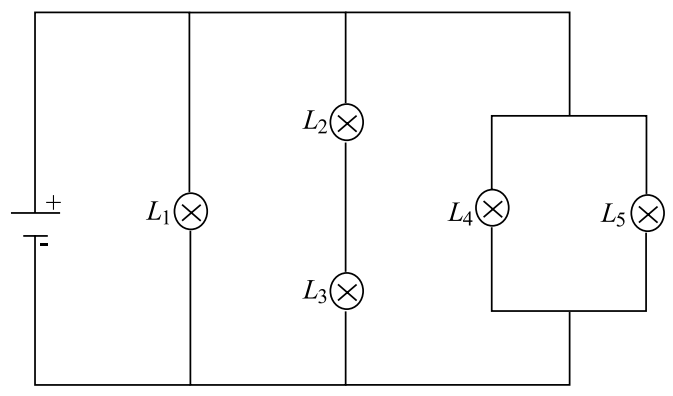

Figura 4 - Teste típico para verificar se os alunos são capazes de classificar as intensidades dos brilhos das lâmpadas em uma associação mista [6].

Muitos alunos apresentam dificuldade para compreender que as divisões de correntes elétricas em um ponto de junção do circuito dependem do que existe em todo o circuito (dificuldade 3.3). Por exemplo, se a resistência elétrica do resistor $R_{1}$, mostrado na Fig. 5, for aumentada, apenas $20 \%$ dos alunos são capazes de responder que $i_{1}$ decrescerá e $i_{2}$ permanecerá constante [5].

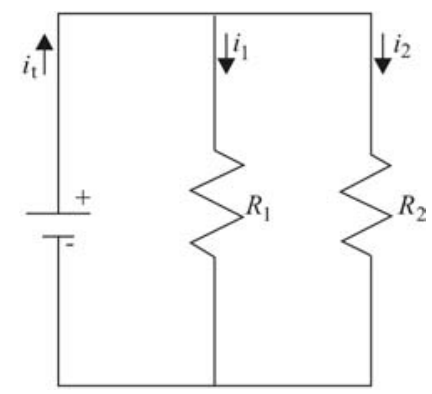

Figura 5 - Teste típico para verificar se os alunos compreendem a divisão de corrente elétrica em uma associação de resistências [5].

d) Concepções alternativas

É bem sabido e divulgado que as concepções alternativas dos alunos influenciam fortemente na aprendizagem. Menos divulgado é o fato de que estas concepções podem influenciar, inclusive o que os alunos vêem. Conforme relatam Schlichting apud Duit e Von Rhöneck [5], é como se os alunos não vissem o que efetivamente devem ver, mas o que as suas concepções lhes permitem ver. Por exemplo, dado o experimento esquematizado na Fig. 6 e questionados sobre em que parte o fio fino incandesceria caso o circuito fosse fechado, os alunos fizeram três diferentes predições: a) primeiro na esquerda (ou direita) dependendo da suposição feita sobre o sentido da corrente e considerando que incandesceria o lado pelo qual a corrente "entra no fio"; b) no meio, já que haveria dois tipos de corrente - uma que entra pela esquerda e outra pela direita - que se encontrariam no meio e c) o fio se incandesceria simultaneamente em todos os lugares (visão correta). Depois das predições terem sido feitas, a experiência foi 
realizada e praticamente todos viram o que esperavam, ou seja, a demonstração apenas reforçou as concepções alternativas dos alunos.

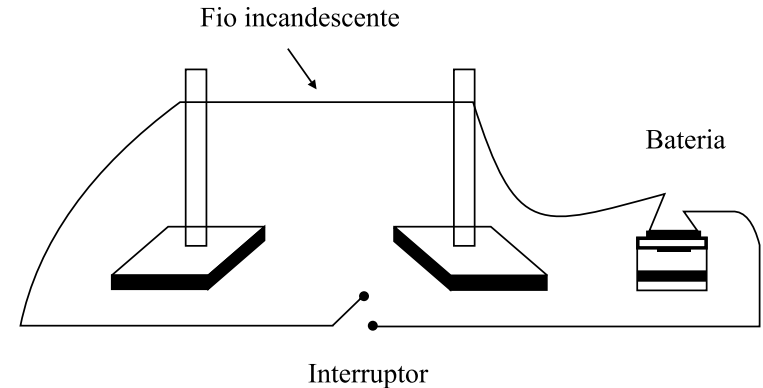

Figura 6 - Esquema do experimento proposto por Schlichting apud Duit \& Von Rhöneck [5] para verificar a influência das concepções alternativas dos alunos.

\subsection{Raciocínios errôneos que os alunos costu- mam apresentar na aprendizagem de cir- cuitos elétricos}

a) O uso de um raciocínio local em vez de sistêmico ou holístico

Muitos alunos focalizam sua atenção em um ponto do circuito e ignoram o que está acontecendo em outra parte. Exemplos de raciocínio local: os alunos consideram a) a bateria como uma fonte de corrente elétrica constante e não como uma fonte de diferença de potencial constante; então, consideram que a corrente elétrica fornecida pela bateria independe do circuito que é conectado à bateria [7] (ver Fig. 1); b) a divisão de corrente elétrica em uma junção do circuito (em associações em paralelo) independe do restante do circuito. Muitos alunos respondem que os valores das intensidades da corrente elétrica no circuito mostrado na Fig. 6 são: $i_{1}=0,6 \mathrm{~A}, i_{2}=0,3 \mathrm{~A}$ e $i_{3}=0,3 \mathrm{~A}[5]$.

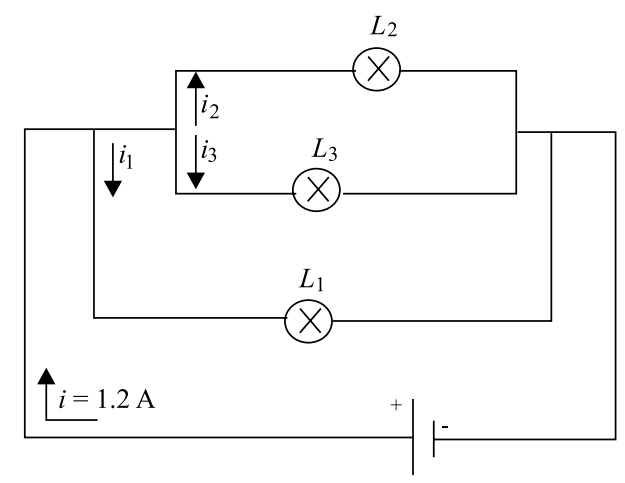

Figura 7 - Representação de um circuito elétrico com lâmpadas associadas em paralelo para verificar a compreensão sobre associação de resistências em paralelo [5].

b) O uso de um raciocínio seqüencial em vez de sistêmico ou holístico

Muitos alunos analisam um circuito elétrico em termos de "antes" e "depois" da passagem de corrente elétrica, isto é, reconhecem que uma mudança no "começo" do circuito influencia os elementos "posteriores", mas consideram que uma mudança no "fim" do circuito não influencia os elementos "anteriores". Na Fig. 8, vê-se um exemplo de tarefa proposta para verificar se o aluno tem raciocínio seqüencial. Em torno de um terço dos alunos avaliados mostraram raciocínio seqüencial [5], i.e., utilizam o raciocínio que se a resistência elétrica $R_{1}$ for alterada, haverá mudança no brilho da lâmpada $L_{1}$, mas se for alterada $R_{2}$ o brilho permanecerá o mesmo em $L_{1}$. Para muitos alunos, por considerarem que o sentido da corrente elétrica e a ordem dos elementos no circuito são relevantes (dificuldade 1.3), se o interruptor for aberto na Fig. 2, o brilho da lâmpada $L_{1}$ permanece constante.

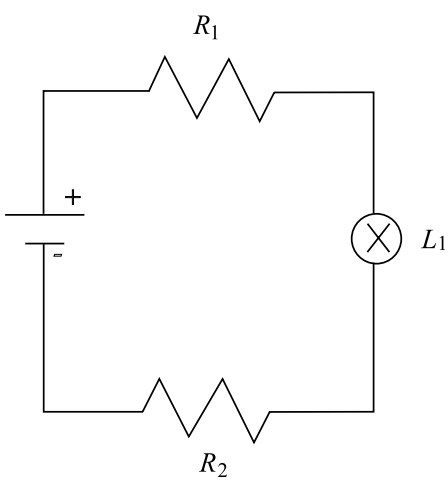

Figura 8 - Testes típicos para verificar se os alunos analisam um circuito elétrico em termos de "antes" e "depois" da passagem de corrente elétrica [5].

\subsection{O uso indiscriminado da linguagem}

Os significados associados pelos alunos a um conceito formal da física são freqüentemente muito diferentes daqueles que um físico atribui a esse mesmo conceito [6]. Em particular, os significados atribuídos aos conceitos de corrente elétrica, diferença de potencial e resistência elétrica na linguagem cotidiana diferem dos significados aceitos cientificamente para estas grandezas. Em outras palavras, o significado atribuído ao conceito de corrente elétrica na linguagem cotidiana inclui um amplo espectro de significados com um certo domínio de idéias sobre energia. Os equívocos em aulas de Física, portanto, são comuns, e mais freqüentes se o professor não estiver ciente destas diferenças entre o seu contexto e o contexto dos alunos para falar sobre fenômenos elétricos [5].

\section{Simulação e modelagem computacio- nais com o software Modellus}

Vários estudos, por exemplo, [12, 13] têm demonstrado que a interatividade do aluno com o material instrucional é um ponto-chave para seu aprendizado. No estudo de circuitos elétricos, a possibilidade mais tradicional de interatividade implica aulas experimentais; porém, 
observa-se que mesmo com o uso sistemático do laboratório [14], a compreensão dos conceitos físicos básicos não atinge o nível desejado. A construção de mapas conceituais e diagramas V (Vê de Gowin) [15] são algumas das técnicas empregadas para promover a interatividade. Propomos outra alternativa, que não exclui as mencionadas: simulação e modelagem computacionais como complemento às atividades em sala de aula e no laboratório de Física ou eletrônica.

Escolhemos como ferramenta para a simulação e modelagem computacionais o software Modellus [16] por permitir que o usuário crie, veja e interaja com representações analíticas, analógicas e gráficas de objetos matemáticos [17]. O Modellus possui uma interface gráfica intuitiva, o que vem a facilitar a interação dos alunos com os modelos em tempo real, permitindo, também, observar múltiplos experimentos (conceituais) simultaneamente. Este software dispensa qualquer conhecimento de linguagem de programação. Na janela Modelo, onde se escreve o modelo matemático subjacente à implementação da atividade computacional, a sintaxe de escrita é praticamente a mesma que se usa ao escrever um modelo no papel. No Modellus uma animação é construída com recursos disponíveis na janela Animação, que podem ser relacionados com variáveis e parâmetros obtidos pela janela Modelo.

A forma como os alunos têm acesso ao modelo matemático ou icônico subjacente à implementação da atividade computacional serve-nos para distinguir entre dois tipos de atividades computacionais, de acordo com os diferentes níveis de interação do aluno com o computador. Em atividades que denominamos de simulação computacional o aluno tem autonomia para inserir valores iniciais para variáveis, alterar parâmetros e, eventualmente, modificar relações entre as variáveis. Entretanto, ele não tem autonomia para modificar o cerne do modelo computacional, ou seja, acesso aos elementos mais básicos, matemáticos ou icônicos, que o constituem. Em atividades que denominamos de modelagem computacional, além de poder atuar sobre a variação de parâmetros e valores iniciais, o aluno tem acesso aos elementos básicos. Em ambos os casos, o aluno pode explorar um modelo computacional já construído, por isto, chama-se este modo de exploratório. No caso da modelagem computacional, o aluno pode, também, construir seu próprio modelo, desde sua estrutura matemática ou icônica até a análise dos resultados gerados por ele, ou fazer alterações em modelos computacionais previamente construídos. Neste caso, diz-se que o modo de uso é expressivo ou de criação.

O material instrucional desenvolvido neste trabalho inclui ambos tipos de atividades (modelagem e simulação) e ambos modos de interação do aluno com o modelo computacional (exploratório e expressivo). Em todas as atividades propostas aos alunos, demo-lhes acesso à janela Modelo do Modellus, mas nem sempre o instigamos a alterá-la. Classificamos como atividade de modelagem toda aquela em que o aluno foi solicitado a trabalhar na janela Modelo, as demais, simulação.

Se instigado com questões apropriadas, que requeiram interação com o modelo, pode-se favorecer a reflexão do aluno sobre os efeitos de suas ações sobre os resultados gerados pelo modelo computacional. Usualmente isto significa que constantemente o aluno está se perguntando: se eu alterar isto, o que acontece com aquilo? As atividades de ensino que propomos visam levá-los a se questionar sobre as relações existentes entre as grandezas básicas de um circuito simples.

\section{Atividades de simulação e modela- gem computacionais}

Nesta seção apresentamos dois exemplos de atividades exploratórias e um exemplo de atividade expressiva. Como mencionado anteriormente, o conjunto completo, com nove atividades, está disponível na Internet [8] e contém, para cada atividade, os objetivos a serem alcançados, os conceitos e raciocínio a serem trabalhados, uma descrição geral do modelo e os enunciados das questões propostas para os alunos (que se encontram nas janelas Notas de cada um dos modelos).

Em nossos modelos representando circuitos elétricos, como usual, admitimos que os resistores são ôhmicos e as fontes e os fios condutores possuem resistência elétrica desprezível.

Uma vez que definimos o foco de nossa atenção em termos das dificuldades conceituais ${ }^{10}$ apresentadas na Tabela 1, estabelecemos os objetivos a serem alcançados pelos alunos ao trabalharem com as atividades de simulação e modelagem computacionais propostas (Tabela 2).

Seguem os três exemplos das atividades computacionais propostas.

Exemplo 1 - Trata-se de uma atividade exploratória, cuja tela principal é apresentada na Fig. 9.

1.1 Objetivos a serem alcançados: objetivos dos itens 1 e 2 apresentados na Tabela 2 .

1.2 Conceitos e raciocínios a serem trabalhados: corrente elétrica, resistência elétrica, diferença de potencial e raciocínio sistêmico.

1.3 Descrição geral do modelo.

A tela principal deste modelo (Fig. 9) apresenta a figura de um circuito elétrico simples composto por:

a) quatro resistores associados em série;

b) uma fonte;

c) quatro voltímetros;

d) uma chave.

10 As dificuldades relacionadas à linguagem não foram explicitamente consideradas, mas permeiam todas as atividades de ensinoaprendizagem que trabalhamos. 


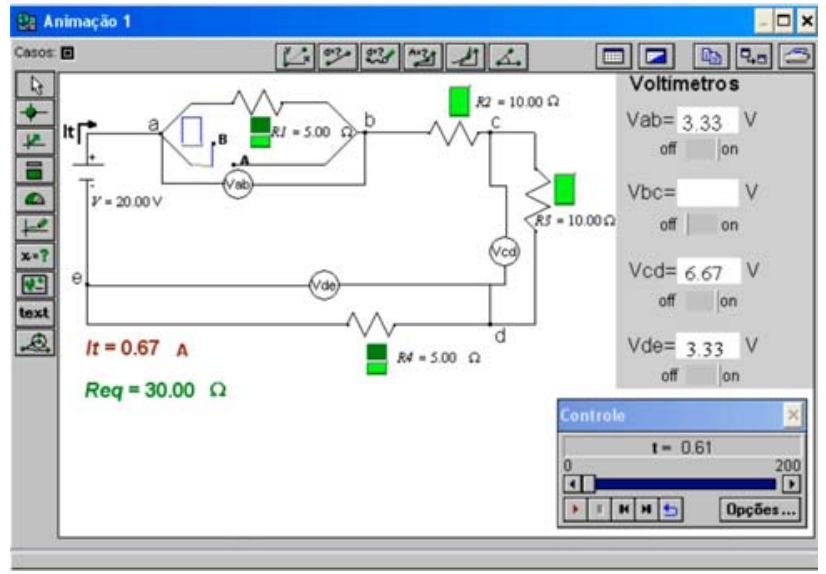

Figura 9 - Janela Animação 1 do modelo cirser.mdl.

Na janela Animação 3 (Fig. 10), ao executar o modelo e mudar a posição da chave, um amperímetro registra a variação da corrente elétrica em um ponto específico do circuito, pelo qual circula a corrente total do circuito $(I t)$.

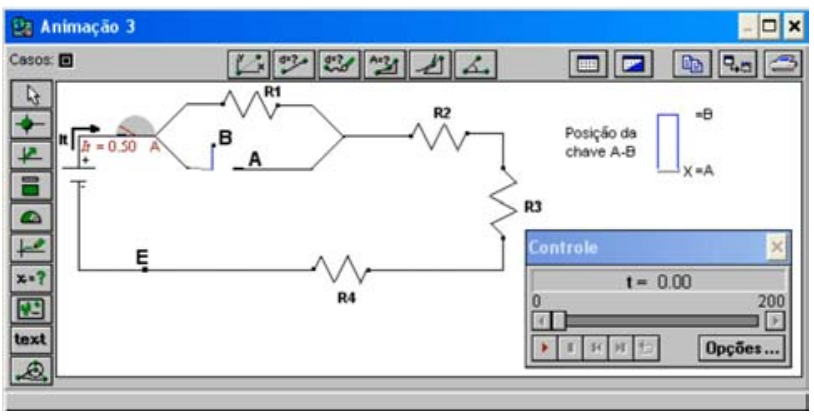

Figura 10 - Janela Animação 3 do modelo cirser.mdl.

Tabela 2 - Objetivos a serem alcançados pelos alunos ao trabalharem com as atividades de simulação e modelagem computacionais sobre circuitos elétricos simples.

\begin{tabular}{|c|c|}
\hline $\operatorname{Dado}(\mathrm{a})$ um(a): & O aluno deverá... \\
\hline $\begin{array}{l}\text { 1. Diferença de potencial } \\
\text { entre pontos de um circuito } \\
\text { simples }\end{array}$ & $\begin{array}{l}\text {...relacionar o aumento da corrente elétrica no circuito à diminuição da resistência equivalente; } \\
\text {...ser capaz de perceber a corrente elétrica como conseqüência da diferença de potencial e da } \\
\text { resistência elétrica. }\end{array}$ \\
\hline $\begin{array}{l}\text { 2. Associação de resistores } \\
\text { em série }\end{array}$ & $\begin{array}{l}\text {...perceber que: } \\
\text { a corrente que circula pelos resistores é a mesma; } \\
\text { a resistência equivalente aumenta quando mais um resistor é associado em série; } \\
\text { a soma das diferenças de potencial entre as extremidades dos resistores é igual à diferença de } \\
\text { potencial aplicada entre os extremos da fonte; } \\
\text { a diferença de potencial aplicada entre os extremos da fonte é característica própria da fonte; } \\
\text { é necessário tratar o circuito elétrico como um sistema. }\end{array}$ \\
\hline $\begin{array}{l}\text { 3. Associação de resistores } \\
\text { em paralelo }\end{array}$ & $\begin{array}{l}\text {...perceber que: } \\
\text { a diferença de potencial entre os extremos dos resistores é a mesma; } \\
\text { a resistência equivalente diminui quando mais um resistor é associado em paralelo; } \\
\text { as divisões de correntes em uma junção do circuito (divisor de corrente) dependem do que } \\
\text { existe no restante do circuito; } \\
\text { a intensidade da corrente elétrica que passa em um resistor depende da sua resistência elétrica } \\
\text { e da diferença de potencial entre os extremos; } \\
\text { é necessário tratar o circuito elétrico como um sistema. }\end{array}$ \\
\hline $\begin{array}{l}\text { 4. Circuito com resistores } \\
\text { em associação mista }\end{array}$ & $\begin{array}{l}\text {...ser capaz de: } \\
\text { tratar o circuito como um sistema; } \\
\text { identificar o comportamento da diferença de potencial nas extremidades dos resistores; } \\
\text { identificar qual é a intensidade de corrente elétrica que passa em cada resistor. }\end{array}$ \\
\hline 5. Circuito com lâmpadas & $\begin{array}{l}\text {...ser capaz de: } \\
\text { identificar que a lâmpada é um resistor e, portanto, os comportamentos observados em relação } \\
\text { a resistores se manifestam também com lâmpadas (itens } 2,3 \text { e 4); } \\
\text { associar o brilho da lâmpada à corrente elétrica. }\end{array}$ \\
\hline $\begin{array}{l}\text { 6. Resistor de resistência } \\
\text { elétrica variável }\end{array}$ & $\begin{array}{l}\text {...perceber as relações existentes entre: } \\
\text { parâmetros geométricos do resistor e resistência elétrica; } \\
\text { resistência elétrica e resistividade. }\end{array}$ \\
\hline
\end{tabular}

Na janela Animação 2 (Fig. 11), com o auxílio dos amperímetros, é possível observar o comportamento da corrente elétrica ao longo do circuito e na Animação 1 (Fig. 9), com o auxílio dos voltímetros, é possível observar a diferença de potencial para os vários pares de pontos ao longo do circuito.

1.4 Enunciado das questões propostas aos alunos

a) Execute o modelo e responda a seguinte questão: ao fechar a chave A-B, o que acontece com a resistência equivalente do circuito mostrado na Animação 3? Por quê?

b) A intensidade da corrente elétrica no ponto E, em relação à intensidade medida no amperímetro, é:

i) menor

ii) igual

iii) maior 


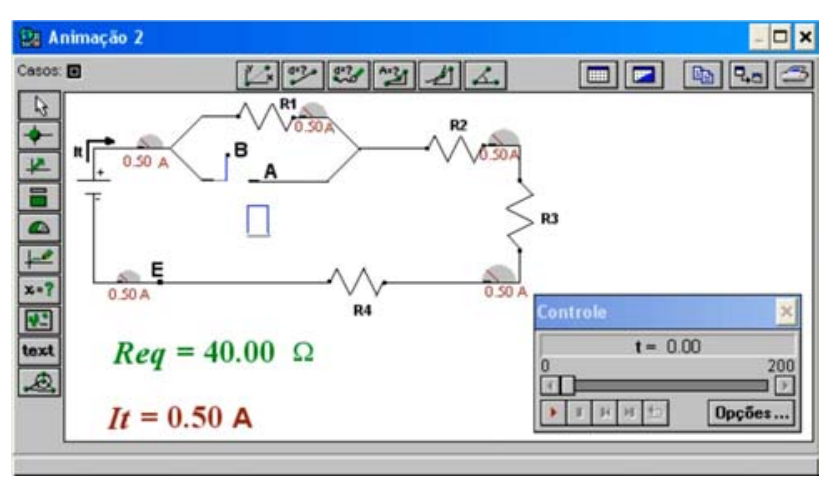

Figura 11 - Janela Animação 2 do modelo cirser.mdl.

c) Abra a Animação 2 (janela $\rightarrow$ Animação 2). Verifique as respostas anteriores. O que se pode dizer sobre o comportamento da corrente elétrica ao longo do circuito?

d) Abra a Animação 1. No lado direito da janela Animação, encontram-se voltímetros capazes de medir diferenças de potencial entre diferentes pares de pontos do circuito. Altere o valor da resistência elétrica nos resistores (através das respectivas barras de rolagem próxima aos mesmos). Quando a chave A-B está na posição $\mathrm{B}$ e os resistores $R_{2}$ e $R_{3}$ nos seus valores máximos, qual é a diferença de potencial entre os pontos b-d?

e) Quando a chave A-B está na posição A e os resistores $R_{2}$ e $R_{3}$ nos seus valores mínimos, qual é a diferença de potencial entre os pontos a-d?

f) Quando a chave A-B está na posição B, clique com o botão esquerdo do mouse em cima do medidor analógico de $R_{4}$ e informe o valor 1000 (valor em ohms). Para que valores tendem a corrente elétrica e a diferença de potencial entre os pontos d-e? Por quê?

g) É possível alterar o valor da diferença de potencial $(V)$ entre os extremos da fonte qualquer que seja a posição da chave A-B? Por quê?

Exemplo 2 - Trata-se de uma atividade exploratória, cuja tela principal é apresentada na Fig. 12.

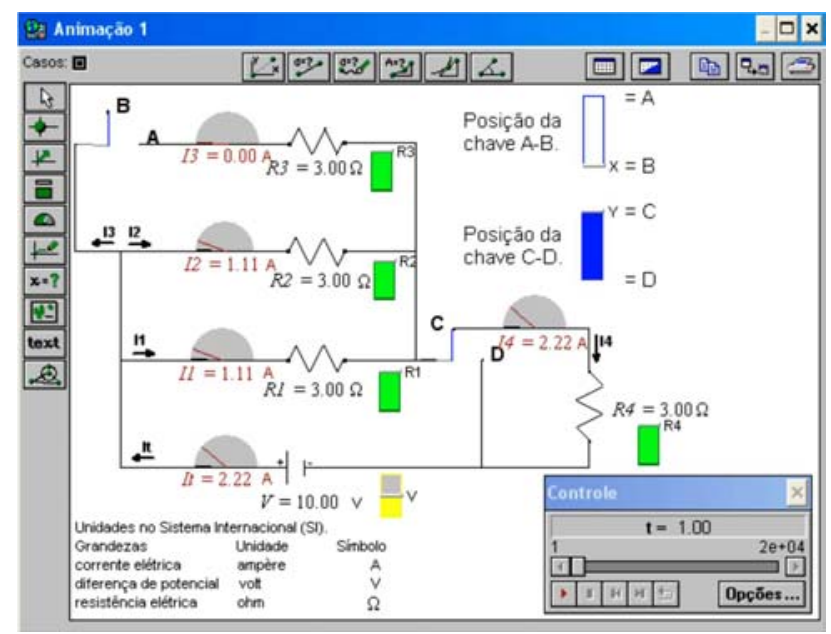

Figura 12 - Janela Animação 1 do modelo cirmis.mdl.
2.1 Objetivos a serem alcançados: objetivos do item 4 apresentado na Tabela 2.

2.2 Conceitos e raciocínio a serem trabalhados: diferença de potencial, corrente elétrica e raciocínio sistêmico.

\subsection{Descrição geral do modelo}

Ao executar o modelo, variando a posição das chaves, torna-se possível obter dois ou três resistores associados em paralelo. Há ainda a possibilidade de associálos em série com um quarto resistor. Os amperímetros registram a corrente elétrica que passa em cada resistor em qualquer posição das chaves. Pode-se alterar a diferença de potencial entre os extremos da fonte e o valor da resistência elétrica nos resistores (através das respectivas barras de rolagem próxima aos mesmos), como é mostrado na Fig. 12.

\subsection{Enunciado das questões propostas aos alunos}

a) Com as chaves nas posições $\mathrm{A}$ e D, altere o valor de $R_{3}$. Explique o comportamento dos valores de $i_{1}, i_{2} \mathrm{e}$ $i_{t}$.

b) Com as chaves nas posições A e C, altere novamente $R_{3}$. Explique porque neste caso todas as correntes variam.

Exemplo 3 - Trata-se de uma atividade expressiva sobre resistência elétrica.

3.1 Objetivos a serem alcançados: objetivos do item 6 apresentado na Tabela 2.

3.2 Conceitos e raciocínio a serem trabalhados: resistência elétrica e resistividade.

\subsection{Descrição geral do modelo}

Nesta atividade propomos aos alunos a construção de um modelo computacional que represente o comportamento da resistência elétrica em um resistor em função da resistividade e de parâmetros geométricos. As Figs. 13 e 14 ilustram um modelo desenvolvido pelos alunos.

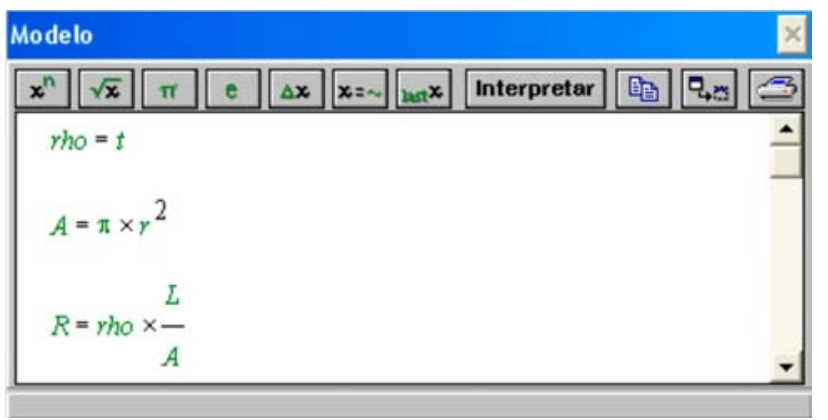

Figura 13 - Janela Modelo de um modelo desenvolvido pelos alunos. 


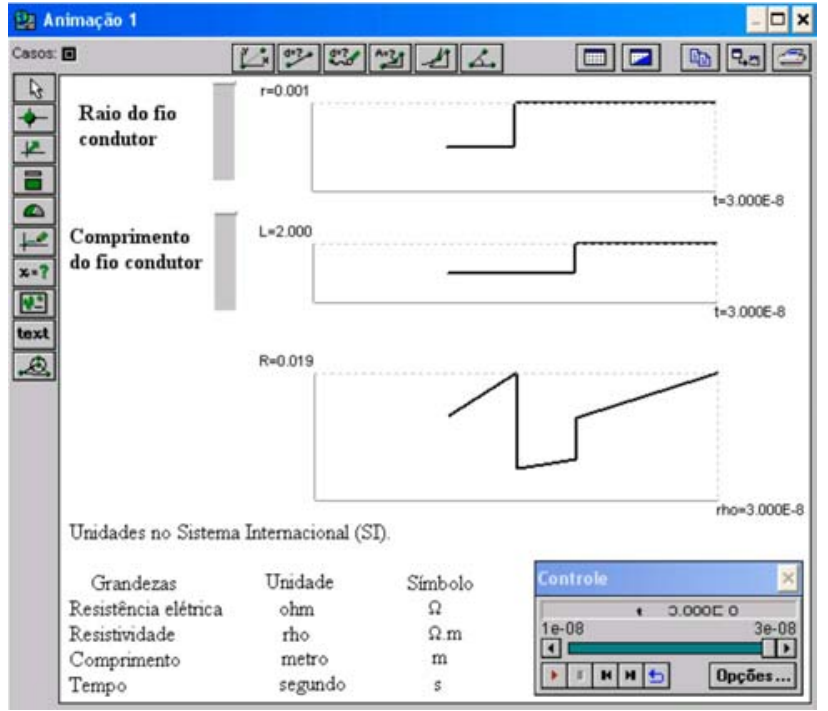

Figura 14 - Janela Animação 1 de um modelo desenvolvido pelos alunos.

3.4 Enunciado das questões propostas aos alunos

Crie um modelo que explore a resistência elétrica em função da resistividade $(\rho)$ e de parâmetros geométricos, de modo que através dele, se possa investigar as relações existentes entre estes conceitos físicos para diversas condições iniciais.

\section{Estratégia de ensino e resultados de uma experiência didática}

O conjunto de atividades computacionais proposto foi utilizado em uma experiência didática como complemento às atividades em sala de aula e no laboratório de Física, durante o horário de aula, nas turmas K e KK da disciplina de Física II-C (FIS01082, Eletricidade e Magnetismo para alunos de Engenharia) oferecida pelo Departamento de Física da UFRGS, no $2^{\circ}$ semestre de 2004. Nossa estratégia de ensino sobre circuitos elétricos simples envolveu, além das atividades computacionais, desenvolvidas no laboratório de informática, um método colaborativo presencial [9] e o método POE (Predizer, Observar, Explicar) [10].

No método colaborativo presencial, o aluno tem uma participação ativa em todas as aulas. Após uma exposição inicial do professor nas aulas introdutórias dos assuntos, tipicamente de 30 a $40 \mathrm{~min}$, o aluno realiza em pequenos grupos, constituídos por dois ou três alunos, alguma tarefa com o auxílio do professor e de monitor(es), quando há. As tarefas realizadas sistematicamente são entregues ao professor ao final de cada aula para fins de avaliação e devolvidas com os comentários pertinentes na aula seguinte. Este modo de avaliação ao longo de toda a disciplina, a participação atuante do aluno em todas as aulas, a mediação feita pelo professor entre os materiais instrucionais e os alunos, e o estabelecimento de relações interpessoais entre o professor e a turma são imprescindíveis para a eficácia do método colaborativo presencial. Neste método, o professor apresenta aos alunos as idéias mais gerais e mais inclusivas da matéria de ensino, durante a exposição inicial, para serem progressivamente diferenciadas em termos de detalhes e especificidades. Este princípio programático da matéria de ensino está ancorado na diferenciação progressiva proposta por Ausubel [1]. Segundo ele: $i$ ) é mais fácil para seres humanos captar aspectos diferenciados de um todo mais inclusivo previamente aprendido, do que chegar ao todo a partir de suas partes diferenciadas previamente aprendidas; ii) a organização do conteúdo de uma certa disciplina, na mente de um indivíduo, é uma estrutura hierárquica na qual as idéias mais inclusivas e gerais estão no topo e, progressivamente, incorporam proposições, conceitos e fatos menos inclusivos e mais diferenciados [2].

Procuramos instigar os alunos a pensar e interagir de modo consciente com os modelos computacionais e não meramente por tentativa e erro. Para isso, baseados no método POE [10], apresentávamos perguntas que deveriam ser respondidas antes de qualquer interação dos alunos com a simulação ou modelo computacional. Por exemplo: inicialmente se solicitava que os alunos fizessem previsões sobre o comportamento da corrente elétrica e da diferença de potencial entre pares de pontos ao longo de um circuito com associação mista de resistores. Então, lhes era dada a oportunidade de manipular a resistência elétrica dos resistores para que observassem se suas previsões seriam confirmadas. Caso isto não ocorresse, lhes era solicitado que explicassem as discrepâncias.

Cabe ressaltar que tanto nas atividades de modelagem quanto nas atividades de simulação, a interação do aluno com o computador era mediada pelo professor tanto em termos de auxílio técnico para a operação do software, como também no esclarecimento de eventuais dúvidas sobre a Física e a Matemática envolvidas no desenvolvimento de seus modelos. As atividades eram desenvolvidas por pequenos grupos de alunos, dispostos em duplas ou trios, para promover não somente a interação dos alunos com as atividades computacionais, mas também entre os próprios alunos.

$\mathrm{Na}$ experiência didática realizada verificou-se que o desempenho (em termos de domínio de situações problemáticas) de alunos que desenvolveram atividades de modelagem e simulação computacionais acrescidas de nossa estratégia de ensino foi superior, de forma estatisticamente significativa, ao desempenho de alunos expostos apenas às tradicionais aulas expositivas e de laboratório [18]. Nossas observações em sala de aula e o levantamento de opiniões dos alunos sugerem que houve muita interação dos alunos com as atividades computacionais, dos alunos entre si e com o professor, tornandose um elemento motivador na aprendizagem dos alunos. A predisposição para aprender é uma das condições da aprendizagem significativa. A outra é que o material seja potencialmente significativo. Acreditamos que 
neste estudo as duas condições foram alcançadas. Para Ausubel [1], um material é dito potencialmente significativo se for relacionável (ou incorporável) à estrutura cognitiva do aluno, de maneira não-arbitrária e não-literal. Esta condição implica que o material deve ser suficientemente não-arbitrário em si, de modo que possa ser aprendido e deve ter relação com a estrutura cognitiva do aluno. Como as atividades computacionais foram propostas com base nas concepções alternativas, dificuldades e raciocínios dos alunos, reportados da literatura, entendemos que o conjunto de atividades propostas é potencialmente significativo.

\section{Comentários gerais}

Nos dias atuais, estudos que identificam dificuldades dos alunos na aprendizagem de um determinado conteúdo de Física não têm se restringido somente à detecção das dificuldades, mas também apresentam concomitantemente alguma alternativa que os auxilie a superá-las. Neste trabalho, levamos em conta as conhecidas dificuldades de aprendizagem enfrentadas pelos alunos na aprendizagem de circuitos elétricos, para conceber um conjunto de atividades de simulação e modelagem computacionais que visam auxiliar os alunos a superá-las. Iniciamos apresentando as dificuldades de aprendizagem detectadas na literatura, não somente porque elas serviram de base para o nosso trabalho, mas porque este artigo também é dirigido a professores. Para Ausubel, segundo Moreira [2]: o fator que mais influencia a aprendizagem é aquilo que o aluno já sabe (cabe ao professor identificar isso e ensinar de acordo). Logo, um aprendizado significativo tem maiores chances de ocorrer quando o professor tem ciência das concepções alternativas, dificuldades conceituais e raciocínios errôneos que seus alunos costumam apresentar. Posteriormente ilustramos algumas das atividades de simulação e modelagem computacionais propostas e disponibilizadas na Internet [8]. Cabe ainda ressaltar que o material instrucional apresentado no presente artigo por si só não garante bons resultados, nossa estratégia de ensino está intimamente relacionada ao sucesso da aprendizagem. Por isto, além de apresentar o material desenvolvido, apresentamos a estratégia de ensino adotada. Finalizamos salientando que em nossa proposta didática o computador não substitui as atividades experimentais, mas, pelo contrário, acrescenta outras situações para que o aluno explore os conteúdos em questão.

\section{Agradecimentos}

Agradecemos ao Prof. Dr. Marco Antonio Moreira que orientou a investigação realizada no âmbito deste trabalho de pesquisa, e ao árbitro pelas relevantes sugestões. Trabalho parcialmente financiado pelo CNPq.

\section{Referências}

[1] D.P. Ausubel, Aquisição e Retenção de Conhecimentos: Uma Perspectiva Cognitiva (Plátano, Lisboa, 2003), $226 \mathrm{p}$.

[2] M.A. Moreira, Aprendizaje Significativo: Teoria e Practica (Visor, Madrid, 2000), 101 p.

[3] I.S. Araujo, E.A. Veit e M.A. Moreira, Rev. Bras. Ens. Fis. 26, 179 (2004).

[4] I.S. Araujo e E.A. Veit, Interpretação de Gráficos da Cinemática. Disponível em http://www. if.ufrgs.br/cref/ntef/cinematica. Acesso em 10 de junho de 2005.

[5] R. Duit e C. Von Rhöneck, in Connecting Research in Physics Education with Teacher Education, edited by A. Tiberghien, E.L. Jossem and J. Barajos (International Commission on Physics Education), disponível em http://www.physics.ohiostate.edu/ jossem/ICPE/C2.html. Acesso em 1 de junho de 2005.

[6] P.S. Shaffer and L.C. McDermott, Am. J. Phys. 60, 994 (1992).

[7] P.V. Engelhardt and R. J. Beichner, Am. J. Phys. 72, 98 (2004).

[8] P.F.T. Dorneles, I.S. Araujo e E.A. Veit, Circuitos Elétricos: Atividades de Simulação e Modelagem Computacionais com o Modellus. Disponível em http://www.if.ufrgs.br/cref/ntef/circuitos. Acesso em 10 de junho de 2005.

[9] I.S. Araujo, Simulação e Modelagem Computacionais como Recursos Auxiliares no Ensino de Física Geral. Tese de Doutoramento em Ciências, Instituto de Física, Universidade Federal do Rio Grande do Sul, 2005.

[10] P. K. Tao and R. F. Gunstone, J. Res. Sci Teach. 36, 859 (1999).

[11] P.F.T. Dorneles, Investigação de Ganhos na Aprendizagem de Conceitos Físicos Envolvidos em Circuitos Elétricos por Usuários da Ferramenta Computacional Modellus. Dissertação de Mestrado em Física, Instituto de Física, Universidade Federal do Rio Grande do Sul, Porto Alegre, 2005.

[12] R.J. Beichner, J. Res. Sci. Teach. 27, 803 (1990).

[13] E.F. Redish and J.M. Wilson, Am. J. Phys. 61, 222 (1993).

[14] P.S. Shaffer and L.C. McDermott, Am. J. Phys. 60, 1003 (1992).

[15] M.A. Moreira, Aprendizaje Significativo: Fundamentación Teórica y Estrategias Facilitadoras (UFRGS, Porto Alegre, 2003), 164 p.

[16] V.D. Teodoro, J.P. Vieira and F.C. Clérigo, Modellus, Interactive Modelling with Mathematica (Knowledge Revolution, San Diego, 1997).

[17] V.D. Teodoro, in International CoLos Conference New Network-Based Media in Education (Maribor, Slovenia, 1998), p. 13.

[18] P.F.T. Dorneles, E.A. Veit e M.A. Moreira, in Atas do $V$ Encontro Nacional de Pesquisa em Educação em Ciências (ENPEC), Bauru, 2005. 